\title{
Generalized Quantum Genetic Algorithm of Multidimensional Space
}

\author{
XiaoFeng Zhang, \\ Key Laboratory of JiangXi procince for Image \\ Processing and Pattern Recognition, \\ Information Engineering of NanChang HangKong \\ University \\ NanChang, JiangXi, 330063, China
}

\author{
Wei Miao \\ Key Laboratory of JiangXi procince for Image \\ Processing and Pattern Recognition, \\ Information Engineering of NanChang HangKong \\ University \\ NanChang, JiangXi, 330063, China
}

\author{
GuiFang Sui , \\ Key Laboratory of JiangXi procince for Image \\ Processing and Pattern Recognition, \\ Information Engineering of NanChang HangKong \\ University \\ NanChang, JiangXi, 330063, China
}

\begin{abstract}
Quantum coding plays an important role in quantum genetic algorithm and affects the optimizing efficiency of algorithm, However, there are some defects in existing quantum genetic algorithm: the quantum coding scheme reduces the stability of the algorithm, making the algorithm easily occurs premature convergence and falls into local extremums. Therefore, coding scheme of multi gene chain is extended in a four dimensional space and multidimensional space based on an improved algorithm. Through simulation experiments of function extremum, combine double and triplestranded coding scheme, in accordance with the characteristics of qubits and the normalization condition. Through the experiment, study the effect of multiple genes encoding scheme chain on the performance of the algorithm. The results show that the optimal results of the algorithm can be better and the precision of solution can be higher by increasing the genetic chain. It is an effective strategy to improve the performance of algorithm.
\end{abstract}

Keywords- Quantum Genetic Algorithm; Optimization Algorithm; Quantum Chromosome Coding; Multidimensional Space; Gene Chain

\section{INTRODUCTION}

Quantum genetic algorithm is a new probabilistic optimization algorithm [1,2,3], currently it's also one of the hot research field of computational intelligence [4,5], which combines both classical genetic algorithm and quantum computing from quantum information theory, has been widely used in engineering. Theoretical foundations of quantum genetic algorithm is very weak, but it has a very important significance at application areas $[6,7,8]$.
Quantum coding plays an important role in quantum genetic algorithm, especially in terms of optimization and efficiency of the algorithm. 2006, Li et al proposed a doublestranded quantum genetic algorithm with two gene-encoding chain[9], the probability magnitude of qubit denotes as parallel gene chains of quantum chromosome gene encoding, be used to solving probability optimization problems. The experiments simulation results show that this is an efficient quantum genetic algorithm. 2008, Li et al proposed a threechain quantum genetic algorithm with three gene-encoding chain [10], used the spherical coordinate from unit Bloch sphere denotes the probability magnitude of qubit, which was seen as a gene locus on the quantum chromosome, so that each the quantum chromosome have three parallel gene chain for solving optimization problems, the experimental results show that add gene chain to the quantum encoding increases the search capabilities for solving the optimization problem solution space, avoid premature convergence, improve the rate of quantum genetic algorithm evolution effectively.

From the above literature, we found that increase the gene chain of quantum chromosome coding can improve the ability of the algorithm to solve optimization problems. But to increase the quantum chromosome gene encoding chain, will have some uncertain impact on the performance of the algorithm optimization? In order to verify the conjecture, combined with double and triple-stranded coding scheme, according to the characteristics and normalization condition of qubits, study the promotion of quantum coding , through experiments investigate the effects of multiple gene chain encoding scheme on algorithm performance. 


\section{PROMOTION OF CODING SCHEME}

\section{A. Coding two dimensional Hilbert space}

Quantum Coding generally are based encoding chromosome on Q-bit probability amplitude $(\alpha, \beta)^{T}$, which $|\alpha|^{2}+|\beta|^{2}=1$, Gene locus of qubits can be in the $|0\rangle$ or $|1\rangle$ state,but also in their superposition state, position information of a gene can be used for storage and expression in qubits. As $|\alpha|^{2}$ gradually approach to $|0\rangle$ state, $|\beta|^{2}$ gradually to approximation normal $|1\rangle$ state, make the quantum chromosome to move closer a particular state and the algorithm gradually have stability. Specific coding scheme is as follows:

$$
\left.q_{i}^{t}=\left[\begin{array}{c|c|c|c|}
\cos \theta_{i 1} & \cos \theta_{i 2} & \mathrm{~L} & \cos \theta_{i m} \\
\sin \theta_{i 1} & \sin \theta_{i 2} & \mathrm{~L} & \sin \theta_{i m}
\end{array}\right]\right]
$$

\section{B. Coding Bloch sphere}

In the three dimensional Bloch sphere, a qubit can be described as:

$$
|\varphi\rangle=\cos \frac{\theta}{2}|0\rangle+e^{i \theta} \sin \frac{\theta}{2}|1\rangle
$$

Wherein $\cos \frac{\theta}{2}$ and $e^{i \theta} \sin \frac{\theta}{2} \quad$ are complex, $\left|\cos \frac{\theta}{2}\right|^{2}$ and $\left|e^{i \theta} \sin \frac{\theta}{2}\right|^{2}$ denote the probability of the qubit in the $|0\rangle$ or $|1\rangle$ state, but they also satisfy the following normalization condition:

$$
\left|\cos \frac{\theta}{2}\right|^{2}+\left|e^{i \theta} \sin \frac{\theta}{2}\right|^{2}=1
$$

Complex $\cos \frac{\theta}{2}$ and $e^{i \theta} \sin \frac{\theta}{2}$ satisfy the above formula condition, which are corresponding to the probability amplitude of the any qubit gene bit, so the probability magnitude of the gene bit can be used to express qubit $\left[\cos \frac{\theta}{2}, e^{i \theta} \sin \frac{\theta}{2}\right]^{T}$. Any quantum bits are corresponding to a point in the Bloch sphere, so qubit can use the Bloch spherical coordinates representation for the $|\varphi\rangle=\left[\begin{array}{lll}\cos \varphi \sin \theta & \sin \varphi \sin \theta & \cos \theta\end{array}\right]^{T}$, the unit of the Bloch spherical coordinates can be decomposed by matrix as follow:

$$
\left(\begin{array}{c}
\cos \theta \sin \varphi \\
\sin \theta \sin \varphi \\
\cos \varphi
\end{array}\right)=\left(\begin{array}{c}
\sin \varphi \cos \theta \\
\sin \varphi \sin \theta \\
\cos \varphi
\end{array}\right)=\left(\begin{array}{c}
\sin \varphi\left(\begin{array}{c}
\cos \theta \\
\sin \theta
\end{array}\right) \\
\cos \varphi
\end{array}\right)
$$

Encoding Bloch sphere as:

$$
q_{i}^{t}=\left[\begin{array}{c|c|c|c|c}
\cos \theta_{i 1} \sin \varphi_{i 1} & \ldots & \cos \theta_{i j} \sin \varphi_{i j} & \ldots & \cos \theta_{i m} \sin \varphi_{i m} \\
\sin \theta_{i 1} \sin \varphi_{i 1} & \ldots & \sin \theta_{i j} \sin \varphi_{i j} & \ldots & \sin \theta_{i 1} \sin \varphi_{i m} \\
\cos \varphi_{i 1} & \ldots & \cos \varphi_{i j} & \ldots & \cos \varphi_{i m}
\end{array} \mid\right]
$$

\section{Derivation of the four-dimensional spatial encoding}

Decomposition of the characteristics of qubit and unit Bloch sphere coordinates can be deduce the fourdimensional space unit coordinates matrix is:

$$
\left(\sin \phi\left(\begin{array}{c}
\sin \varphi\left(\begin{array}{c}
\cos \theta \\
\sin \theta
\end{array}\right) \\
\cos \varphi
\end{array}\right)=\cdots=\left(\begin{array}{c}
\sin \phi \sin \varphi \cos \theta \\
\sin \phi \sin \varphi \sin \theta \\
\sin \phi \cos \varphi \\
\cos \phi
\end{array}\right)=\left(\begin{array}{c}
\cos \theta \sin \varphi \sin \phi \\
\sin \theta \sin \varphi \sin \phi \\
\cos \varphi \sin \phi \\
\cos \phi
\end{array}\right)\right.
$$

Quantum encoding of four dimensional on unit space is:

$$
\left.q_{i}^{t}=\left[\begin{array}{c|c|c|c|c}
\cos \theta_{i 1} \sin \varphi_{i 1} \sin \phi_{i 1} & \ldots & \cos \theta_{i j} \sin \varphi_{i j} \sin \phi_{i j} & \ldots & \cos \theta_{i m} \sin \varphi_{i m} \sin \phi_{i m} \\
\sin \theta_{i 1} \sin \varphi_{i 1} \sin \phi_{i 1} & \ldots & \sin \theta_{i j} \sin \varphi_{i j} \sin \phi_{i j} & \ldots & \sin \theta_{i m} \sin \varphi_{i m} \sin \phi_{i m} \\
\cos \varphi_{i 1} \sin \phi_{i 1} & \ldots & \cos \varphi_{i j} \sin \phi_{i j} & \ldots & \cos \varphi_{i m} \sin \phi_{i m} \\
\cos \phi_{i 1} & \ldots & \cos \phi_{i j} & \ldots & \cos \phi_{i m}
\end{array}\right]\right)
$$

\section{The promotion of coding on multi-dimensional space}

The coordinates matrix of the multidimensional space can be obtained by the coordinate matrix of Bloch sphere and four dimensional space as follow:

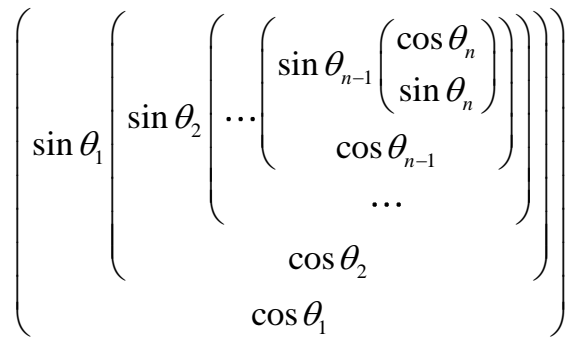

$$
\begin{aligned}
& =\left(\operatorname { s i n } \theta _ { 1 } \left(\sin \theta_{2}\left(\begin{array}{c}
\sin \theta_{n-1} \cos \theta_{n} \\
\left.\cdots\left(\begin{array}{c}
\sin \theta_{n-1} \sin \theta_{n} \\
\cos \theta_{n-1} \\
\ldots
\end{array}\right)\right) \\
\cos \theta_{2} \\
\cos \theta_{1}
\end{array}\right)\right.\right. \\
& =\ldots \ldots=\left(\begin{array}{c}
\cos \theta_{n} * \ldots * \sin \theta_{2} \sin \theta_{1} \\
\sin \theta_{n} * \ldots * \sin \theta_{2} \sin \theta_{1} \\
\vdots \\
\cos \theta_{3} \sin \theta_{2} \sin \theta_{1} \\
\cos \theta_{2} \sin \theta_{1} \\
\cos \theta_{1}
\end{array}\right)
\end{aligned}
$$

Quantum encoding of multi-dimensional unit space is:

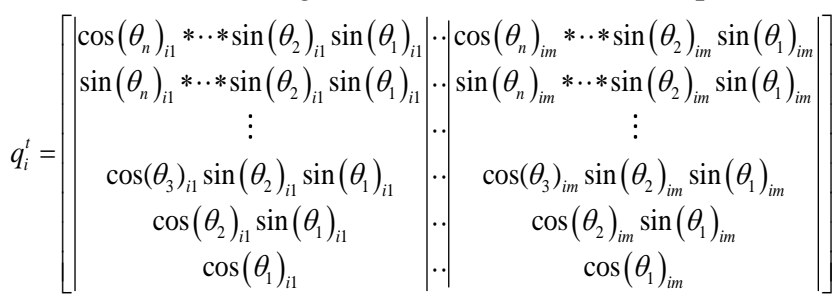




\section{PROMOTION OF THE CONVERSION OF SOLUTION SPACE}

\section{A. The conversion of Hilbert space solution space}

Assume that $X_{j}$ is a variable of optimization problem, It's domain is $\left[a_{i}, b_{i}\right]$, the $\mathrm{j}$-th qubit on the quantum chromosome $\left|q_{i}\right\rangle$ is $\left[\cos \left(\theta_{i j}\right)_{j}^{i}, \sin \left(\theta_{i j}\right)_{j}^{i}\right]^{T}$, So the solution space variables can be expressed by the following formula:

$$
\left[\begin{array}{c}
X_{j 1}^{i} \\
X_{j 2}^{i}
\end{array}\right]=\left[\begin{array}{cc}
\frac{b_{i}-a_{i}}{2} & 0 \\
0 & \frac{b_{i}-a_{i}}{2}
\end{array}\right]\left[\begin{array}{c}
\cos \left(\theta_{i j}\right)_{j}^{i} \\
\sin \left(\theta_{i j}\right)_{j}^{i}
\end{array}\right]+\left[\begin{array}{c}
\frac{b_{i}+a_{i}}{2} \\
\frac{b_{i}+a_{i}}{2}
\end{array}\right]
$$

That is:

$$
\left\{\begin{array}{l}
X_{j 1}^{i}=\frac{1}{2}\left[b_{j}\left(1+\cos \left(\theta_{i j}\right)_{j}^{i}\right)+a_{j}\left(1-\cos \left(\theta_{i j}\right)_{j}^{i}\right)\right] \\
X_{j 2}^{i}=\frac{1}{2}\left[b_{j}\left(1+\sin \left(\theta_{i j}\right)_{j}^{i}\right)+a_{j}\left(1-\sin \left(\theta_{i j}\right)_{j}^{i}\right)\right]
\end{array}\right.
$$

Which $\mathrm{i}=1,2, \cdots, \mathrm{m} ; \mathrm{j}=1,2, \cdots, \mathrm{n}$. For convenience, Set $\left[\cos \left(\theta_{i j}\right)_{j}^{i}, \sin \left(\theta_{i j}\right)_{j}^{i}\right]^{T}=\left[\alpha_{j}^{i}, \beta_{j}^{i}\right]^{T}$, The solution space variables can be expressed as:

$$
\left\{\begin{array}{l}
X_{j 1}^{i}=\frac{1}{2}\left[b_{j}\left(1+\alpha_{j}^{i}\right)+a_{j}\left(1-\alpha_{j}^{i}\right)\right] \\
X_{j 2}^{i}=\frac{1}{2}\left[b_{j}\left(1+\beta_{j}^{i}\right)+a_{j}\left(1-\beta_{j}^{i}\right)\right]
\end{array}\right.
$$

\section{B. The conversion of Bloch sphere solution space}

Assume that $X_{j}$ is a variable of optimization problem, It's domain is $\left[a_{i}, b_{i}\right]$, the $\mathrm{j}$-th qubit on the quantum chromosome $\left|q_{i}\right\rangle$ as follow:

$$
\left[\cos \left(\theta_{i j}\right)_{j}^{i} \sin \left(\varphi_{i j}\right)_{j}^{i}, \sin \left(\theta_{i j}\right)_{j}^{i} \sin \left(\varphi_{i j}\right)_{j}^{i}, \cos \left(\varphi_{i j}\right)_{j}^{i}\right]^{T}
$$

Simplify denoted as: $\left[\beta_{i j}^{1}, \beta_{i j}^{2}, \beta_{i j}^{3}\right]^{T}$, So the solution space variables can be expressed by the following formula:

$$
\left[\begin{array}{l}
X_{j 1}^{i} \\
X_{j 2}^{i} \\
X_{j 3}^{i}
\end{array}\right]=\left[\begin{array}{ccc}
\frac{b_{i}-a_{i}}{2} & 0 & 0 \\
0 & \frac{b_{i}-a_{i}}{2} & 0 \\
0 & 0 & \frac{b_{i}-a_{i}}{2}
\end{array}\right]\left[\begin{array}{c}
\beta_{i j}^{1} \\
\beta_{i j}^{2} \\
\beta_{i j}^{3}
\end{array}\right]+\left[\begin{array}{c}
\frac{b_{i}+a_{i}}{2} \\
\frac{b_{i}+a_{i}}{2} \\
\frac{b_{i}+a_{i}}{2}
\end{array}\right]
$$

Simplify finishing can get:

$$
\left\{\begin{array}{l}
X_{j 1}^{i}=\frac{1}{2}\left[b_{j}\left(1+\beta_{i j}^{1}\right)+a_{j}\left(1-\beta_{i j}^{1}\right)\right] \\
X_{j 1}^{i}=\frac{1}{2}\left[b_{j}\left(1+\beta_{i j}^{2}\right)+a_{j}\left(1-\beta_{i j}^{2}\right)\right] \\
X_{j 1}^{i}=\frac{1}{2}\left[b_{j}\left(1+\beta_{i j}^{3}\right)+a_{j}\left(1-\beta_{i j}^{3}\right)\right]
\end{array}\right.
$$

\section{The promotion of four-dimensional solution space}

Assume that $X_{j}$ is a variable of optimization problem, It's domain is $\left[a_{i}, b_{i}\right]$, the $\mathrm{j}$-th qubit on the quantum chromosome $\left|q_{i}\right\rangle$ as follow:

$$
\left[\begin{array}{c}
\cos \left(\left(\theta_{n}\right)_{i 1}\right)_{j}^{i} * \cdots * \sin \left(\left(\theta_{2}\right)_{i 1}\right)_{j}^{i} \sin \left(\left(\theta_{1}\right)_{i 1}\right)_{j}^{i} \\
\cdots \\
\cos \left(\left(\theta_{2}\right)_{i 1}\right)_{j}^{i} \sin \left(\left(\theta_{1}\right)_{i 1}\right)_{j}^{i} \\
\cos \left(\left(\theta_{1}\right)_{i 1}\right)_{j}^{i}
\end{array}\right]
$$

Simplify denoted as: $\left[\beta_{i j}^{1}, \beta_{i j}^{2}, \beta_{i j}^{3}, \beta_{i j}^{4}\right]^{T}$, So the solution space variables can be expressed by the following formula:

$$
\left\{\begin{array}{l}
X_{j 1}^{i}=\frac{1}{2}\left[b_{j}\left(1+\beta_{i j}^{1}\right)+a_{j}\left(1-\beta_{i j}^{1}\right)\right] \\
X_{j 2}^{i}=\frac{1}{2}\left[b_{j}\left(1+\beta_{i j}^{2}\right)+a_{j}\left(1-\beta_{i j}^{2}\right)\right] \\
X_{j 3}^{i}=\frac{1}{2}\left[b_{j}\left(1+\beta_{i j}^{3}\right)+a_{j}\left(1-\beta_{i j}^{3}\right)\right] \\
X_{j 4}^{i}=\frac{1}{2}\left[b_{j}\left(1+\beta_{i j}^{4}\right)+a_{j}\left(1-\beta_{i j}^{4}\right)\right]
\end{array}\right.
$$

\section{The promotion of multi-dimensional solution space}

Assume that $X_{j}$ is a variable of optimization problem, It's domain is $\left[a_{i}, b_{i}\right]$, the $\mathrm{j}$-th qubit on the quantum chromosome $\left|q_{i}\right\rangle$ as follow:

$$
\left[\begin{array}{c}
\cos \left(\theta_{i j}\right)_{j}^{i} \sin \left(\varphi_{i j}\right)_{j}^{i} \sin \left(\phi_{i j}\right)_{j}^{i} \\
\sin \left(\theta_{i j}\right)_{j}^{i} \sin \left(\varphi_{i j}\right)_{j}^{i} \sin \left(\phi_{i j}\right)_{j}^{i} \\
\cos \left(\varphi_{i j}\right)_{j}^{i} \sin \left(\phi_{i j}\right)_{j}^{i} \\
\cos \left(\phi_{i j}\right)_{j}^{i}
\end{array}\right]
$$

Simplify denoted as: $\left[\beta_{i j}^{1}, \cdots, \beta_{i j}^{n-1}, \beta_{i j}^{n}\right]^{T}$, So the solution space variables can be expressed by the following formula:

$$
\left\{\begin{array}{c}
X_{j 1}^{i}=\frac{1}{2}\left[b_{j}\left(1+\beta_{i j}^{1}\right)+a_{j}\left(1-\beta_{i j}^{1}\right)\right] \\
\ldots \\
X_{j n-1}^{i}=\frac{1}{2}\left[b_{j}\left(1+\beta_{i j}^{n-1}\right)+a_{j}\left(1-\beta_{i j}^{n-1}\right)\right] \\
X_{j n}^{i}=\frac{1}{2}\left[b_{j}\left(1+\beta_{i j}^{n}\right)+a_{j}\left(1-\beta_{i j}^{n}\right)\right]
\end{array}\right.
$$




\section{PROMOTION OF QUANTUM ROTATION GATE}

A. Quantum rotation gate of two dimensional Hilbert space

Quantum rotation gate of quantum phase on two dimensional Hilbert space is:

$$
U=\left[\begin{array}{cc}
\cos \Delta \theta & -\sin \Delta \theta \\
\sin \Delta \theta & \cos \Delta \theta
\end{array}\right]
$$

The update process is:

$$
U\left[\begin{array}{c}
\cos \theta \\
\sin \theta
\end{array}\right]=\left[\begin{array}{c}
\cos (\theta+\Delta \theta) \\
\sin (\theta+\Delta \theta)
\end{array}\right]
$$

\section{B. Quantum rotation gate of Bloch sphere}

Quantum rotation gate of quantum phase on Bloch sphere is:

$$
U=\left[\begin{array}{ccc}
\cos \Delta \varphi \cos \Delta \theta & -\sin \Delta \varphi \cos \Delta \theta & \sin \Delta \theta \cos (\varphi+\Delta \varphi) \\
\sin \Delta \varphi \cos \Delta \theta & \cos \Delta \varphi \cos \Delta \theta & \sin \Delta \theta \sin (\varphi+\Delta \varphi) \\
-\sin \Delta \theta & -\tan (\varphi / 2) \sin \Delta \theta & \cos \Delta \theta
\end{array}\right]
$$

The update process is:

$$
U\left[\begin{array}{c}
\cos \varphi \sin \theta \\
\sin \varphi \sin \theta \\
\cos \theta
\end{array}\right]=\left[\begin{array}{c}
\cos (\varphi+\Delta \varphi) \sin (\theta+\Delta \theta) \\
\sin (\varphi+\Delta \varphi) \sin (\theta+\Delta \theta) \\
\cos (\theta+\Delta \theta)
\end{array}\right]
$$

C. The promotion of Quantum rotation gate on fourdimensional space

The results of quantum rotation gate of quantum phase on four-dimensional space is more complicated, not list here.

The update process is:

$$
U\left(\begin{array}{c}
\cos \theta \sin \varphi \sin \phi \\
\sin \theta \sin \varphi \sin \phi \\
\cos \varphi \sin \phi \\
\cos \phi
\end{array}\right)=\left(\begin{array}{c}
\cos (\theta+\Delta \theta) \sin (\varphi+\Delta \varphi) \sin (\phi+\Delta \phi) \\
\sin (\theta+\Delta \theta) \sin (\varphi+\Delta \varphi) \sin (\phi+\Delta \phi) \\
\cos (\varphi+\Delta \varphi) \sin (\phi+\Delta \phi) \\
\cos (\phi+\Delta \phi)
\end{array}\right)
$$

D. The promotion of Quantum rotation gate on multidimensional space

The results of quantum rotation gate of quantum phase on multi-dimensional space is more complicated, not list here. The update process is:

$$
U\left(\begin{array}{c}
\cos \theta_{n} * \ldots * \sin \theta_{2} \sin \theta_{1} \\
\sin \theta_{n} * \ldots * \sin \theta_{2} \sin \theta_{1} \\
\vdots \\
\cos \theta_{3} \sin \theta_{2} \sin \theta_{1} \\
\cos \theta_{2} \sin \theta_{1} \\
\cos \theta_{1}
\end{array}\right)=\left(\begin{array}{c}
\cos \left(\theta_{n}+\Delta \theta_{n}\right) * \cdots * \sin \left(\theta_{2}+\Delta \theta_{2}\right) \sin \left(\theta_{1}+\Delta \theta_{1}\right) \\
\sin \left(\theta_{n}+\Delta \theta_{n}\right) * \cdots * \sin \left(\theta_{2}+\Delta \theta_{2}\right) \sin \left(\theta_{1}+\Delta \theta_{1}\right) \\
\vdots \\
\cos \left(\theta_{3}+\Delta \theta_{3}\right) \sin \left(\theta_{2}+\Delta \theta_{2}\right) \sin \left(\theta_{1}+\Delta \theta_{1}\right) \\
\cos \left(\theta_{2}+\Delta \theta_{2}\right) \sin \left(\theta_{1}+\Delta \theta_{1}\right) \\
\cos \left(\theta_{1}+\Delta \theta_{1}\right)
\end{array}\right)
$$

We can use the method of undetermined coefficients for solving quantum rotation gate of multidimensional space, the solving process and results is complex, not list here.

Principles of quantum chromosome mutation and the above is same, as it no longer details here.

\section{SIMULATIION AND ANALYSIS OF THE EXPERIMENTAL RESULTS}

A. The simulation

Through Bohachevsky and Goldstein-Price function extreme optimization and simulation functions, further validate conjectures of multi-chain optimization results.

Experimental conditions: personal PC, software: Matlab.

1) Bohachevsky function and Goldstein-Price function

Bohachevsky function as follow:

$$
\begin{array}{r}
f_{1}(x, y)=0.3 \cos 3 \pi x-0.3 \cos 4 \pi y-x^{2}-y^{2}-0.3 \\
(x, y) \in(-1,1)
\end{array}
$$

This function has an unlimited number of local maxima, of which only two is the largest global, Symmetrically at $(0$,$0.23)$ and $(0,0.23)$, Global maximum is 0.24003441039434 . If the result of the optimization algorithm is larger than 0.235 , the convergence of the algorithm is considered. Fig .1 for this function.

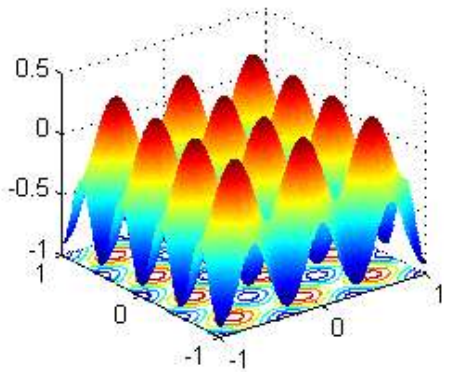

Figure 1. Function $\mathrm{f}_{1}(\mathrm{x}, \mathrm{y})$ graph

Goldstein-Price function as follow:

$$
\begin{array}{r}
f_{2}(x, y)=\left[1+(x+y+1)^{2}\left(19-14 x+3 x^{2}-14 y+6 x y+3 y^{2}\right)\right] \\
*\left[30+(2 x-3 y)^{2}\left(18-32 x+12 x^{2}+48 y-36 x y+27 y^{2}\right)\right] \\
(x, y) \in[-2,2]
\end{array}
$$

This function has four minima:(1.2,0.8),(1.2,0.2),(-0.6,$0.4),(0,-1)$. Global minimum point is $(0,-1)$, Global minimum value is 3 . When the optimization result is less than 3.005 , the convergence can be considered. Fig .2 for this function.

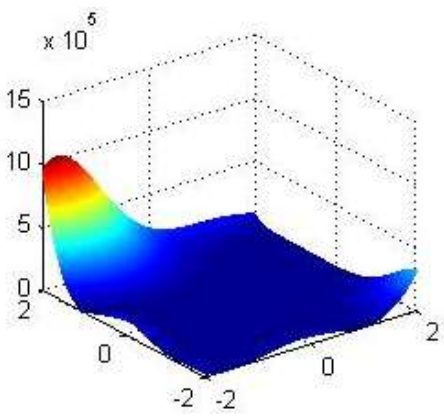

Figure 2. Function $\mathrm{f}_{2}(\mathrm{x}, \mathrm{y})$ graph

\section{2) Simulation}

For each of the two functions were optimized 10 with duplexes coding, triple-stranded coding, and quadruplex coding. 
Setting algorithm parameters are as follows: population size is 50; quantum bits is 2; probability of gene mutation is 0.1 ; angle step is set to the initial value $0.001 \pi$; the evolution algebra is 500. optimization results of function $\mathrm{f}_{1}$ $(\mathrm{x}, \mathrm{y})$ and $\mathrm{f}_{2}(\mathrm{x}, \mathrm{y})$ shown in Fig .3 respectively.
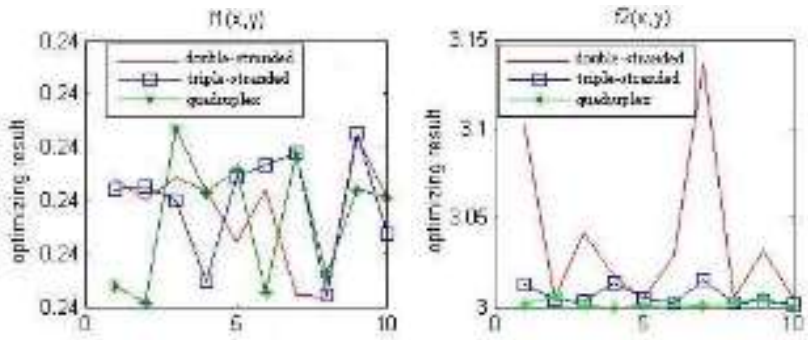

Figure 3. Optimization results of function $\mathrm{f}_{1}(\mathrm{x}, \mathrm{y})$ and $\mathrm{f}_{2}(\mathrm{x}, \mathrm{y})$

Function $\mathrm{f}_{1}(\mathrm{x}, \mathrm{y})$ corresponding to the optimal result and the convergence curve as shown in Fig .4 .
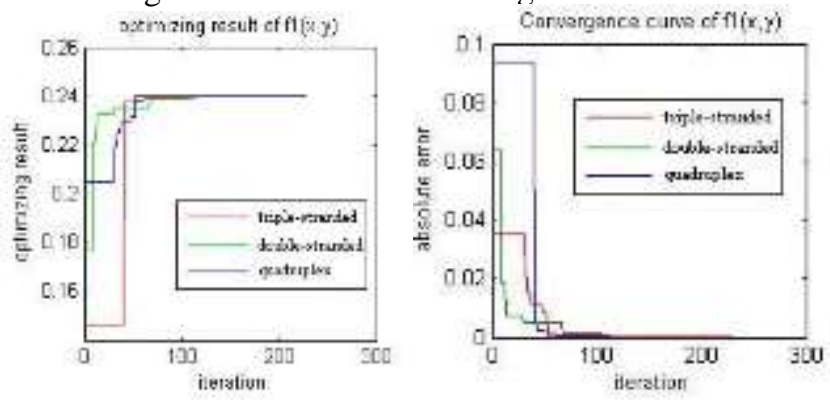

Figure 4. Optimal result and convergence curve of function $f_{1}(x, y)$

Function $\mathrm{f}_{2}(\mathrm{x}, \mathrm{y})$ corresponding to the optimal result and the convergence curve as shown in Fig .5.
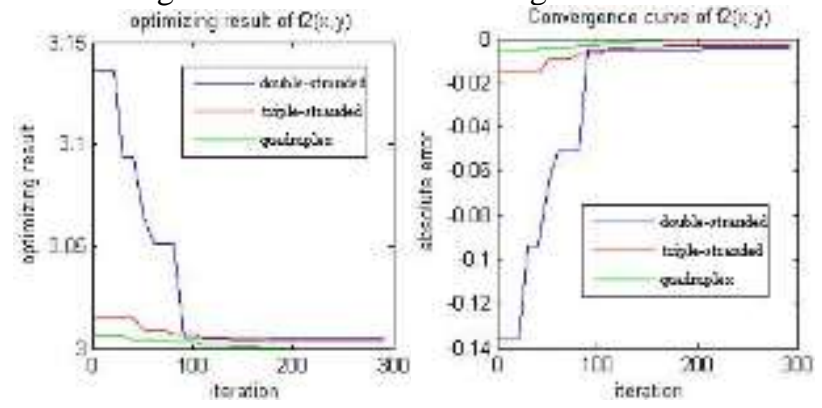

Figure 5. Optimal result and convergence curve of function $\mathrm{f}_{2}(\mathrm{x}, \mathrm{y})$

Function $\mathrm{f}_{1}(\mathrm{x}, \mathrm{y})$ and $\mathrm{f}_{2}(\mathrm{x}, \mathrm{y})$ comparative optimization results shown in Table 1.

\section{B. Analysis}

We can be seen from Table 1, optimal results of quantum genetic algorithm quadruplex coding higher than triplestranded and double-stranded, convergence number is the same situation.
TABLE I. COMPARATIVE OPTIMIZATION RESULTS OF FUNCTION $\mathrm{F}_{1}(\mathrm{X}$, Y) $\operatorname{AND~F}_{2}(\mathrm{X}, \mathrm{Y})$

\begin{tabular}{|c|c|c|c|c|c|c|}
\hline $\begin{array}{c}\text { Coding } \\
\text { scheme }\end{array}$ & \multicolumn{3}{|c|}{$f_{1}(x, y)$} & \multicolumn{3}{c|}{$f_{2}(x, y)$} \\
\cline { 2 - 7 } & $\begin{array}{c}\text { Double } \\
\text { Strand } \\
\text {-ed }\end{array}$ & $\begin{array}{c}\text { Triple } \\
\text { Strand } \\
\text {-ed }\end{array}$ & $\begin{array}{c}\text { Quadr- } \\
\text { uplex }\end{array}$ & $\begin{array}{c}\text { Double } \\
\text { Strand } \\
\text {-ed }\end{array}$ & $\begin{array}{c}\text { Triple } \\
\text { Strand } \\
\text {-ed }\end{array}$ & $\begin{array}{c}\text { Quadr- } \\
\text { uplex }\end{array}$ \\
\hline $\begin{array}{c}\text { Optimal } \\
\text { results }\end{array}$ & $\begin{array}{c}0.2400 \\
31\end{array}$ & $\begin{array}{c}0.2400 \\
32\end{array}$ & $\begin{array}{c}0.2400 \\
33\end{array}$ & 3.0047 & 3.0030 & 3.0000 \\
\hline $\begin{array}{c}\text { Worst } \\
\text { results }\end{array}$ & $\begin{array}{c}0.2400 \\
019\end{array}$ & $\begin{array}{c}0.2400 \\
022\end{array}$ & $\begin{array}{c}0.2400 \\
026\end{array}$ & 3.1360 & 3.0152 & 3.0059 \\
\hline $\begin{array}{c}\text { Average } \\
\text { results }\end{array}$ & $\begin{array}{c}0.2400 \\
22\end{array}$ & $\begin{array}{c}0.2400 \\
24\end{array}$ & $\begin{array}{c}0.2400 \\
25\end{array}$ & 3.0418 & 3.0070 & 3.0016 \\
\hline $\begin{array}{c}\text { Converg } \\
\text { ence } \\
\text { times }\end{array}$ & 8 & 8 & 9 & 4 & 9 & 9 \\
\hline $\begin{array}{c}\text { Average } \\
\text { time(s) }\end{array}$ & 4.870 & 5.275 & 10.74 & 1.3126 & 2.2693 & 2.4042 \\
\hline $\begin{array}{c}\text { Average } \\
\text { number } \\
\text { of steps }\end{array}$ & 106 & 151 & 228 & 112 & 136 & 182 \\
\hline
\end{tabular}

Due to an increase in the gene strand, the conversion of the solution space will be complexity and increase the magnitude of the phase angle of quantum rotation gate slower, make the corresponding increase in running time(running time can be negligible).

In short, by increasing the genetic chain allows the algorithm to search capacity for the solution space increased, greatly increase the search space, while optimizing the accuracy of the algorithm has been significantly improved, avoid premature convergence of the algorithm appears. It's an effective strategy to improve the efficiency of the optimization algorithm.

\section{CONCLUSIONS}

This paper focuses on the promote of quantum coding scheme, appropriate to promote the solution space conversion, quantum rotation gate, quantum chromosome mutation, and through Bohachevsky function and GoldsteinPrice function extreme optimization to simulation. Experimental results show that: increased gene chain is an effective strategy to improve the efficiency of the algorithm optimization. Further research should study the complexity of the algorithm.

\section{ACKNOWLEDGEMENT}

This work was financially supported by the Natural Science Foundation of China (No.60973048), Research Foundation of Jiangxi Province Education Department (No.GJJ12413), and Key Laboratory Open Foundation (No.TX201204003).

\section{REFERENCES}

[1] TALBI H,DRAA A,BATOUCHE M.A. new quantum-inspired genetic algorithm for solving the travelling salesman problem[C]. Proceedings of the International Conference on Industrial Technology, 2004, 3:1192-1197.

[2] HAN K H,KIM J H.Quantum-inspired evolutionary algorithm for a class of combinational optimization[J].IEEE Trans Evolutionary Computation, 2002, 6(6): 1354-1360.

[3] K H Han, J H Kim. Genetic quantum algorithm and its application to combinational optimization problem[C]. Proceedings of the International Congress on Evolutionary Computation. IEEE Press.2000:1354-1360. 
[4] Qian Jie, Zheng Jianguo, Zhang Chaoqiu. Review of the status quo Algorithm Quantum evolution [J]. Control and Decision, 2011,26 (3) :321-331. (in Chinese)

[5] Gexiang Zhang. Quantum-inspired evolutionary algorithms: a survey and empirical study [J]. Journal of Heuristics,2011,3(17):303-351.

[6] P. Arpaiaa, D.Maistob, C.Mannac. A Quantum-inspired Evolutionary Algorithm with a competitive variation operator for Multiple-Fault Diagnosis [J]. Applied Soft Computing, 2011: 4655-4566.

[7] Mani A, Patvardhan C. Solving ceramic grinding optimization problem by adaptive quantum evolutionary algorithm[C]. 2010 Int Conf on Intelligent Systems Modeling and Simulation. Berk, 2010:43-48.

[8] De Pinho Curz. A quantum inspired neuro evolutionary algorithm with binary real representation[C]. 2009 World Congress on Nature and Biologically Inspired Computing. NaBIC,2009:445-450.

[9] LI.PC,LI.SY. Based on real-coded and the objective function gradient quantum genetic algorithm [J]. Harbin Institute of Technology,2006, 38(8):1216-1219.

[10] LI.PC,LI.SY.Quantum-inspired evolutionary algorithm for continuous spaces optimization [J]. Chinese Journal of Electronics, 2008,17(1):80-84 . 